\title{
Une remarque sur les solutions bornées d'une équation différentielle avec pilotage
}

\author{
par Z. Mikolauska (Kraków)
}

À la mémoire de Franciszek Leja

Résumé. Dans la note présente nous allons démontrer une condition suffisante pour l'existence d'un pilotage borné $\left\{u_{1}=\varphi_{1}(t), u_{2}=\varphi_{2}(t)\right\}$ tel que chaque solution $y(t)$ de l'équation $y^{\prime}=f\left(t, y, \varphi_{1}(t), \varphi_{2}(t)\right)$ avec la condition initiale $|y(0)|<k$ reste bornée pour $t \in[0, \infty)$.

Envisageons l'équation différentielle

$$
y^{\prime}(t)=f\left(t, y(t), u_{1}, u_{2}\right)
$$

où $f\left(t, y, u_{1}, u_{2}\right)$ est de classe $C^{1}$ par rapport à $\left(t, y, u_{1}, u_{2}\right)$. Nous allons donner une condition suffisante pour l'existence d'un pilotage borné $u_{1}=\varphi_{1}(t), \quad u_{2}=\varphi_{2}(t)$ tel que chaque solution $\left(y(t), \varphi_{1}(t), \varphi_{2}(t)\right)$ de l'équation (1) avec la condition initiale

$$
|y(0)|<k
$$

reste bornée pour $t \in[0, \infty)$.

Remarque. Il est évident qu'il suffit de donner des conditions sur $f\left(t, y, u_{1}, u_{2}\right)$ sous lesquelles il existe un pilotage $\left(\varphi_{1}(t), \varphi_{2}(t)\right)$ tel que

$$
\begin{aligned}
f\left(t, k, \varphi_{1}(t), \varphi_{2}(t)\right)<0 & \text { pour } 0 \leqslant t<\infty, \\
f\left(t,-k, \varphi_{1}(t), \varphi_{2}(t)\right)>0 & \text { pour } 0 \leqslant t .
\end{aligned}
$$

1. Hypotheses H. Supposons que

$$
\left|\begin{array}{ll}
i_{u_{1}}(t, k, u), & f_{u_{2}}(t, k, u) \\
f_{u_{1}}(t,-k, u), & f_{u_{2}}(t,-k, u)
\end{array}\right|>0
$$

pour $0 \leqslant t<\infty,\|u\| \leqslant R(R>0)$

$$
f(0, k, 0,0)<0, \quad f(0,-k, 0,0)>0,
$$




$$
\begin{aligned}
& f_{t}(t, k, u)\left\{f_{u_{1}}(t,-k, u) u_{2}-f_{u_{2}}(t,-k, u) u_{1}\right\}- \\
& -f_{t}(t,-k, u)\left\{f_{u_{1}}(t, k, u) u_{2}-f_{u_{2}}(t, k, u) u_{1}\right\}<0 \\
& \text { pour } R>\|u\|>0 .
\end{aligned}
$$

THÉoRÈmE T. Les hypothèses $\dot{H}$ étant admises:

(I) il existe une constante $r>0, r \leqslant R$, telle que chaque solution du système

$$
\begin{aligned}
f\left(t, k, u_{1}, u_{2}\right) & =f\left(0, k, u_{1}^{0}, u_{2}^{0}\right), \\
f\left(t,-k, u_{1}, u_{2}\right) & =f\left(0,-k, u_{1}^{0}, u_{2}^{0}\right),
\end{aligned}
$$

avec $\left\|u^{0}\right\| \leqslant r$ et avec la condition initiale $\varphi_{1}(0)=u_{1}^{0}, \varphi_{2}(0)=u_{2}^{0}$ existe pour $0 \leqslant t<\infty$ et satisfait à rinégalité

$$
\|\varphi(t)\|<r \quad \text { pour } 0 \leqslant t<\infty .
$$

(II) Pour le pilotage $u_{1}=\varphi_{1}(t), u_{2}=\varphi_{2}(t)$ ainsi défini sont satisfaites les inégalités (3), c'est-à-dire que chaque solution $y(t)$ de réquation (1) avec la condition (2) et avec le pilotage $\varphi(t)$ satisfaisant à (1.4) reste bornée pour $0 \leqslant t<\infty$ et

$$
|y(t)|<k \quad \text { pour } 0 \leqslant t<\infty .
$$

Démonstration. De la continuité de $f(t, y, u)$ il résulte qu'il existe une constante $r>0$ telle que

$$
\begin{aligned}
f\left(0, k, u_{1}, u_{2}\right)<0 & \text { pour }\|u\| \leqslant r, \\
f\left(0,-k, u_{1}, u_{2}\right)>0 & \text { pour }\|u\| \leqslant r .
\end{aligned}
$$

Envisageons $\left(u_{1}^{0}, u_{2}^{0}\right)$ tel que $\left\|u^{0}\right\| \leqslant r$ et la solution $\varphi_{1}(t), \varphi_{2}(t)$ du système d'équations différentielles

$$
\begin{aligned}
\varphi_{1}^{\prime}(t) & =\frac{-f_{t}(t, k, \varphi) f_{u_{2}}(t,-k, \varphi)+f_{t}(t,-k, \varphi) f_{u_{2}}(t, k, \varphi)}{f_{u_{1}}(t, k, \varphi) f_{u_{2}}(t,-k, \varphi)-f_{u_{2}}(t, k, \varphi) f_{u_{1}}(t,-k, \varphi)}, \\
\varphi_{2}^{\prime}(t) & =\frac{-f_{1}(t,-k, \varphi) f_{u_{1}}(t, k, \varphi)+f_{t}(t, k, \varphi) f_{u_{1}}(t,-k, \varphi)}{f_{u_{1}}(t, k, \varphi) f_{u_{2}}(t,-k, \varphi)-f_{u_{2}}(t, k, \varphi) f_{u_{1}}(t,-k, \varphi)}
\end{aligned}
$$

avec la condition initiale

$$
\varphi_{1}(0)=u_{1}^{0}, \quad \varphi_{2}(0)=u_{2}^{0} .
$$

Envisageons la fonction composée

$$
f\left(t, k, \varphi_{1}(t), \varphi_{2}(t)\right) \stackrel{d f}{=} \sigma_{1}(t), \quad f\left(t,-k, \varphi_{1}(t), \varphi_{2}(t)\right) \stackrel{d f}{=} \sigma_{2}(t) .
$$

On a $\sigma_{1}(0)<0, \sigma_{2}(0)>0$

$$
\begin{aligned}
& \sigma_{1}^{\prime}(t)=f_{t}(t, k, \varphi)+f_{u_{1}}(t, k, \varphi) \varphi_{1}^{\prime}+f_{u_{2}}(t, k, \varphi) \varphi_{2}^{\prime}, \\
& \sigma_{2}^{\prime}(t)=f_{t}(t,-k, \varphi)+f_{u_{1}}(t,-k, \varphi) \varphi_{1}^{\prime}+f_{u_{2}}(t,-k, \varphi) \varphi_{2}^{\prime} .
\end{aligned}
$$


Posons par définition

$$
D(t)=\left|\begin{array}{ll}
f_{u_{1}}(t, k, \varphi), & f_{u_{2}}(t, k, \varphi) \\
f_{u_{1}}(t,-k, \varphi), & f_{u_{2}}(t,-k, \varphi)
\end{array}\right| .
$$

On a donc

$$
\begin{aligned}
\sigma_{1}^{\prime}(t)= & \frac{1}{D(t)}\left\{f_{t}(t, k, \varphi) f_{u_{1}}(t, k, \varphi) f_{u_{2}}(t,-k, \varphi)-\right. \\
& -f_{t}(t, k, \varphi) f_{u_{2}}(t, k, \varphi) f_{u_{1}}(t,-k, \varphi)- \\
& -f_{u_{1}}(t, k, \varphi) f_{t}(t, k, \varphi) f_{u_{2}}(t,-k, \varphi)+ \\
& +f_{u_{1}}(t, k, \varphi) f_{t}(t,-k, \varphi) f_{u_{2}}(t, k, \varphi)- \\
& -f_{u_{2}}(t, k, \varphi) f_{t}(t,-k, \varphi) f_{u_{1}}(t, k, \varphi)+ \\
& \left.+f_{u_{2}}(t, k, \varphi) f_{t}(t, k, \varphi) f_{u_{1}}(t,-k, \varphi)\right\}=0,
\end{aligned}
$$

d'où il vient

$$
\gamma_{1}(t)=f\left(t, k, \varphi_{1}(t), \varphi_{2}(t)\right)=\text { const }=f\left(0, k, u^{0}\right)<0 .
$$

D'une façon analogue on obtient

$$
\sigma_{2}(t)=f\left(t,-k, \varphi_{1}(t), \varphi_{2}(t)\right)=\text { const }=f\left(0,-k, u^{0}\right)>0 .
$$

Ainsi nous avons démontré que la solution $\left(\varphi_{1}, \varphi_{2}\right)$ du système (1.7) avec la condition (1.8) satisfait à (1.4) et à (3). Il rèste à prouver que le pilotage $u_{1}=\varphi_{1}(t), u_{2}=\varphi_{2}(t)$ est défini dans $[0, \infty)$ et satisfait à (1.5). Supposons que $r \leqslant R$. On a donc $D(t)>0$ pour $t \geqslant 0$ tels que $\|\varphi\| \leqslant r$. On a

$$
\begin{aligned}
\frac{1}{2}\left(\varphi_{1}^{2}+\varphi_{2}^{2}\right)^{\prime}= & \varphi_{1} \varphi_{1}^{\prime}+\varphi_{2} \varphi_{2}^{\prime} \\
= & \frac{1}{D(t)}\left\{-f_{t}(t, k, \varphi) f_{u_{2}}(t,-k, \varphi) \varphi_{1}+\right. \\
& +f_{t}(t,-k, \varphi) f_{u_{2}}(t, k, \varphi) \varphi_{1}- \\
& \left.-f_{t}(t,-k, \varphi) f_{u_{1}}(t, k, \varphi) \varphi_{2}+f_{t}(t, k, \varphi) f_{u_{1}}(t,-k, \varphi) \varphi_{2}\right\} \\
= & \frac{1}{D(t)}\left\{f_{t}(t, k, \varphi)\left[f_{u_{1}}(t,-k, \varphi) \varphi_{2}-f_{u_{2}}(t,-k, \varphi) \varphi_{1}\right]-\right. \\
& \left.-f_{t}(t-k, \varphi)\left[f_{u_{1}}(t, k, \varphi) \varphi_{2}-f_{u_{2}}(t, k, \varphi) \varphi_{1}\right]\right\} .
\end{aligned}
$$

En vertu de (1.3) on a donc

$$
\frac{1}{2}\left\{\varphi_{1}^{2}+\varphi_{2}^{2}\right\}^{\prime}<0 \quad \text { pour } t \geqslant 0 \text { tel que }\|\varphi\| \leqslant r, 0 \leqslant t<\infty,
$$

et par suite $\|\varphi\|<r$ pour $0 \leqslant t<\infty$.

Le théorème $\mathrm{T}$ est ainsi démontré. Dans la suite allons donner un simple exemple de l'application du théorème $T$. 
2 Exemple E. Envisageons le cas où

$$
f\left(t, y, u_{1}, u_{2}\right) \stackrel{d f}{=} a(t)\left[(y-\alpha) u_{1}+(y-\beta) u_{2}\right]-y,
$$

(2.2) $a(t)$ est une fonction de classe $C^{1}$ pour $0 \leqslant t<\infty$,

$$
a(t) \cdot a^{\prime}(t)>0 \text { pour } 0 \leqslant t<x,
$$

(2.4) les constantes $x, \beta$ satisfont à l'inégalité $\beta<\alpha$.

Dans le cas envisagé on a

$$
\begin{aligned}
& \left|\begin{array}{l}
f_{u_{1}}\left(t, k, u_{1}, u_{2}\right), \quad f_{u_{2}}\left(t, k, u_{1}, u_{2}\right) \\
f_{u_{1}}\left(t,-k, u_{1}, u_{2}\right), f_{u_{2}}\left(t,-k, u_{1}, u_{2}\right)
\end{array}\right| \\
& =a^{2}(t)\left|\begin{array}{ll}
k-\alpha, & k-\beta \\
-k-\alpha, & -k-\beta
\end{array}\right|=-a^{2}(t)[(k-\alpha)(k+\beta)-(k-\beta)(k+\beta)] \\
& =-a^{2}(t)\left[k^{2}+(\beta-x) k-x \beta-k^{2}+(\beta-\alpha) k+\alpha \beta\right] \\
& =-a^{2}(t) \cdot 2(\beta-x) k>0
\end{aligned}
$$

c'est-à-dire que (1.1) est satisfaite. Envisageons $f(0, k, 0,0)$ et $f(0,-k, 0,0)$. On a $f(0, k, 0,0)=-k<0, f(0,-k, 0,0)=k>0$. C'est-à-dire que (1.2) est satisfaite. Démontrons (1.3)

$$
\begin{aligned}
& f_{t}\left(t, k, u_{1}, u_{2}\right)\left[f_{u_{1}}\left(t,-k, u_{1}, u_{2}\right) u_{2}-\right. \\
&\left.-f_{u_{2}}\left(t,-k, u_{1}, u_{2}\right)\left(t,-k, u_{1}, u_{2}\right) u_{1}\right]- \\
&-f_{1}\left(t,-k, u_{1}, u_{2}\right)\left[f_{u_{1}}\left(t, k, u_{1}, u_{2}\right) u_{2}-f_{u_{2}}\left(t, k, u_{1}, u_{2}\right) u_{1}\right] \\
&= 2 a^{\prime}(t) \cdot a(t)(\beta-\alpha)\left(u_{1}^{2}+u_{2}^{2}\right)<0 \quad \text { pour } u_{1}^{2}+u_{2}^{2}>0
\end{aligned}
$$

(cf. (2.2), (2.3), (2.4)). On peut donc appliquer le théorème $T$. Dans le cas envisagé on peut facilement évaluer $r$. On vérifie aisémant que $r$ doit satisfaire à l'inégalité suivante:

$$
0<r<\min \left\{\frac{k}{|a(0)|[|k-\alpha|+|k-\beta|]}, \frac{k}{|a(0)|[|k+\alpha|+|k+\beta|]}\right\} .
$$

Dans le cas où la condition (2.5) est satisfaite, on a

$$
\begin{aligned}
f\left(0, k, u_{1}, u_{2}\right) & =a(0)\left[(k-\alpha) u_{1}+(k-\beta) u_{2}\right]-k \\
& \leqslant|a(0)|[|k-\alpha|+|k-\beta|] r-k<0, \\
f\left(0,-k, u_{1}, u_{2}\right) & =-a(0)\left[(k+\alpha) u_{1}+(k+\beta) u_{2}\right]+k \\
& \geqslant-|a(0)|[|k+\alpha|+|k+\beta|] r+k>0 .
\end{aligned}
$$

